\title{
CONVENIENT ONE-POT SYNTHESIS OF 2-CARBAMOYLMETHYLTHIO- 3-CYANO-4,6-DIARYL-5-ETHOXYCARBONYL-1,4-DIHYDROPYRIDINES
}

\author{
A.Krauze*, L.Sĩle, G.Duburs \\ Latvian Institute of Organic Synthesis, Riga, Aizkraukles 21, LV-1006, Latvia; krauzęr.s: .iv
}

\begin{abstract}
Carbamoylmethylthio-3-cyano-4,6-diaryl-5-ethoxycarbonyl-1,4-dihydropyridines $\underline{1}$ were obtained by an one-pot condensation of ethyl 4-nitrobenzoylacetate, an aromatic aldehyde and cyanothioacetamide in the presence of piperidine with subsequent alkylation and dehydroxylation. Thorpe's cyclization of $\underline{1}$ yielded thieno[2,3-b]pyridines $\underline{\mathbf{3}}$.
\end{abstract}

\section{Introduction}

2-Alkylthio-3-cyano-1,4-dihydropyridines, which have been obtained by alkylation of 1,4dihydropyridine-2-thiones $[1,2]$, have revealed cardiovascular $[3,4]$, antioxidant and hepatoprotective $[5,6]$ activities. The corresponding 2-alkylthio-3-cyano-6-hydroxy-1,4,5,6tetrahydropyridines exhibit tangible cardiovascular activity too [3], but, unfortunately, these compounds without strong electron withdrawing group at 5 position and sterically bulky group at 6 position are characterized as unstable compounds. They in course of preparation split off water molecule to give 2-alkylthio-1,4- or 4,5-dihydropyridines $[7,8]$.

In continuation of searching biologically active compounds we have elabolarated convenient one-pot synthesis of 2-carbamoylmethylthio-3-cyano-4,6-diaryl-5-ethoxycarbonyl-1,4-dihydropyridines $\underline{1}$ and investigated their Thorpe's cyclization.

\section{Results and discussion}

Alkylation of 1,4-dihydropyridine-2(3H)-thiones bearing three nucleophilic reaction centres $(\mathrm{S}$, $1-\mathrm{N}$ and 3-C) at mild reaction conditions preceeded exclusively at sulphur atom [1,2]. Synthesis of 1,4-dihydropyridine-2(3H)-thiones bearing electron donating substituents in position 4 is rather complicated because they, being good antioxidants [9], in course of preparation and isolation oxidize to corresponding pyridine-2(1H)-thiones. 
Convenient method of one-pot synthesis of 2-carbamoylmethylthio-3-cyano-4,6-diaryl-5ethoxycarbonyl-1,4-dihydropyridines 1 (also bearing electron donating substituents in position 4) has been elaborated by condensation of ethyl 4-nitrobenzoylacetate, an aromatic aldehyde and cyanothioacetamide in the presence of piperidine with subsequent treatment with iodoacetamide and acidification. The yields were $64-70 \%$, only in case of $1 \mathrm{c}$ it was $86 \%$.

Piperidinium 6-hydroxy-1,4,5,6-tetrahydropyridine-2-thiolates as primary intermediates have been isolated only in case of 4-phenyl substituent [10]. To carry out a one-pot condensation of ethyl 4-nitrobenzoylacetate, an aromatic aldehyde and cyanothioacetamide in the presence of piperidine with subsequent alkylation (without acidification) only in case of 4-phenyl substituent 2carbamoylmethylthio-1,4,5,6-tetrahydropyridine $\underline{\mathbf{2}}$ has been obtained. 2-carbamoylmethylthio-1,4dihydropyridine 1 a was prepared also in $95 \%$ yield by short heating of 2-çarbamoylmethylthio1,4,5,6-tetrahydropyridine $\underline{2}$ in $\mathrm{HCl}$-ethanol solution.

Treatment of $\underline{\mathbf{1 a}-\mathbf{d}}$ with $\mathrm{KOH}$ in water-ethanol solution gave thieno[2,3-b]pyridines $\underline{\mathbf{3 a}-\mathbf{d}}$ (62 $80 \%$ yields) which means that next to the Thorpe's cyclization spontaneous oxidation of dihydropyridine cycle took place.

The structures of synthesized compounds were proved by spectroscopic methods. In the IR spectra characteristic absorbtion bands of $3-\mathrm{C}=\mathrm{N}$ group for dihydropyridines $\underline{1}$ and tetrahydropyridine $\underline{2}$ at $2194-2204 \mathrm{~cm}^{-1}$ are observed, which disappeared by Thorpe's cyclization (in case of compounds 3 ). Absorbtion bands of $v \mathrm{C}=\mathrm{O}$ of compounds are in agreement with the type of conjugation of $\mathrm{C}=\mathrm{O}$ groups. The doublets in the case of ${ }^{1} \mathrm{H} N \mathrm{NR}$ spectrum of $\underline{\mathbf{2}}$ with $\mathrm{J}_{4,5}=12 \mathrm{~Hz}$ according to [7] confirm a trans-diaxial configuration of the $4-\mathrm{H}$ and $5-\mathrm{H}$ protons. In case of 1,4dihydropyridines $\underline{1}$, the characteristic $4-\mathrm{H}$ proton signals at $4.56-4.80 \mathrm{ppm}$ are observed

In conclusion a convenient method of one-pot synthesis of 2-carbamoylmethylthio-3-cyano-4,6diaryl-5-ethoxycarbonyl-1,4-dihydropyridines $\underline{1}$ have been elaborated by condensation of ethyl 4nitrobenzoylacetate, an aromatic aldehyde and cyanothioacetamide in the presence of piperidine with subsequent treatment with iodoacetamide and acidification. 2-Carbamoylmethyllthio-3-cyano6-hydroxy-1,4,5,6-tetrahydropyridine $\underline{\underline{2}}$ as intermediate is isolated. The Thorpe's cyclization of $\underline{1}$ is supported by spontaneous oxidation of dihydropyridine cycle. 


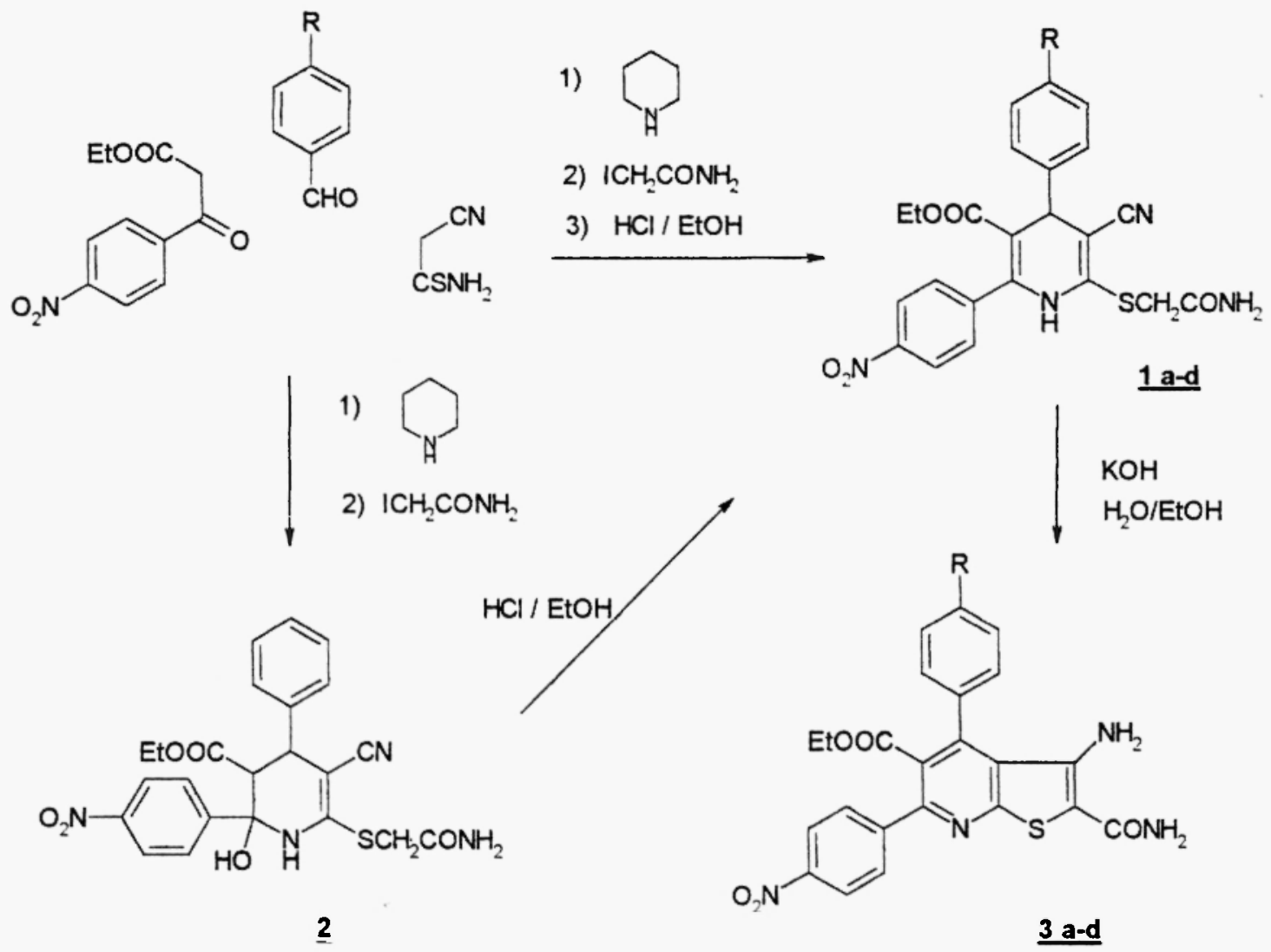
a) $\mathbf{R}=\mathbf{H}$;
b) $\mathrm{R}=\mathrm{NO}_{2}$; c) $\mathrm{R}=\mathrm{OMe}$;
d) $\mathrm{R}=\mathrm{OH}$

\section{Experimental}

Melting points were determined on a Boetius apparatus and are uncorrected. IR spectra were recorded on a Perkin-Elmer $580 \mathrm{~B}$ spectrometer (in nujol) and peak positions $v_{\max }$ were expressed in $\mathrm{cm}^{-1}$. ${ }^{1} \mathrm{H}$ NMR spectra were recorded on a Bruker WH-90 spectrometer. Chemical shifts are expressed in $\delta$ (p.p.m. downfield from TMS) and coupling constants $(J)$ in Hertz. The course of the reactions and the individuality of substances were monitored by TLC on Kieselgel $60 \mathrm{~F}$ Merck plates with dichloromethane - hexane - methanol $(5: 5: 1)$ as eluent. Compounds were recrystallized from ethanol.

\section{2-Carbamoylmethylthio-3-cyano-5-ethoxycarbonyl-6-(4-nitrophenyl)-4-phenyl-1,4-dihydro-}

pyridine 1a. A mixture of ethyl 4-nitrobenzoylacetate $(1.19 \mathrm{~g}, 5 \mathrm{mmol})$, benzaldehyde $(0.53 \mathrm{~g}, 5$ $\mathrm{mmol})$, cyanothioacetamide $(0.5 \mathrm{~g}, 5 \mathrm{mmol})$ and piperidine $(0.55 \mathrm{ml}, 5.5 \mathrm{mmol})$ in $25 \mathrm{ml}$ of ethanol was shortly heated until dissolution and stirred for $10 \mathrm{~min}$ at ambient temperature. Then to the reaction mixture iodoacetamide ( $1.02 \mathrm{~g}, 5.5 \mathrm{mmol})$ was added, shortly heated and stirred for $15 \mathrm{~min}$ 
at the ambient temperature. Finally $5 \mathrm{ml}$ of $3 \mathrm{~N}$ hydrochloric acid in ethanol was added, refluxed for $5 \mathrm{~min}$ and stirred for $1 \mathrm{~h}$ at the ambient temperature. The precipitate was filtered and washed with $10 \mathrm{ml}$ of ethanol, $10 \mathrm{ml}$ of water to give $1.55 \mathrm{~g} \mathrm{(67 \% )} \mathrm{of} 1 \mathrm{a}$ as yellow crystals; $\mathrm{mp} 208$ $210^{\circ} \mathrm{C}$. IR: 1680, $1708(\mathrm{C}=\mathrm{O}), 2204(\mathrm{C} \equiv \mathrm{N}) ; 3190,3362\left(\mathrm{NH}, \mathrm{NH}_{2}\right) ;{ }^{1} \mathrm{H}$ NMR (DMSO-d $\left.\mathrm{d}_{6}\right): 0.75$ $\left(3 \mathrm{H}, \mathrm{t}, \mathrm{CH}_{2} \mathrm{CH}_{3}\right) ; 3.65$ and $3.90\left(1 \mathrm{H}, \mathrm{d}\right.$ and $\left.\mathrm{d}, \mathrm{J}=15 \mathrm{~Hz}, \mathrm{SCH}_{2}\right) ; 3.75\left(2 \mathrm{H}, \mathrm{q}, \mathrm{CH}_{2} \mathrm{CH}_{3}\right) ; 4.66(1 \mathrm{H}$, s, 4-H); 7,3 - $7.4\left(5 \mathrm{H}, \mathrm{m}, 4-\mathrm{C}_{6} \mathrm{H}_{5}\right) ; 7.64$ and $7.96\left(2 \mathrm{H}\right.$, br. s and br. s, $\left.\mathrm{CONH}_{2}\right), 7.75$ and $8.28(4 \mathrm{H}$, $\mathrm{d}$ and $\left.\mathrm{d}, 6-\mathrm{C}_{6} \mathrm{H}_{4}\right) ; 10.95(1 \mathrm{H}, \mathrm{s}, \mathrm{NH})$. Calcd for $\mathrm{C}_{23} \mathrm{H}_{20} \mathrm{~N}_{4} \mathrm{O}_{5} \mathrm{~S}: \mathrm{C} 59.47, \mathrm{H} 4.51, \mathrm{~N}$ 12.06, S 6.90. Found C 59.54, H 4.37, N 11.95, S 6.90.

In a similar manner (4-nitrobenzaldehyde was used instead of benzaldehyde) 2carbamoylmethylthio-3-cyano-4,6-di-(4-nitrophenvl)-5-ethoxycarbonyl-1,4-dihydropyridine $\underline{\text { 1b }}$ was obtained $(86 \%)$ as slightly yellow powder, mp $203-205^{\circ} \mathrm{C}$; IR: 1664,1680 sh, 1696 $(\mathrm{C}=\mathrm{O}) ; 2194(\mathrm{C} \equiv \mathrm{N}) ; 3200,3262,3340,3412\left(\mathrm{NH}, \mathrm{NH}_{2}\right)$; ${ }^{1} \mathrm{H}$ NMR (DMSO- $\left.\mathrm{d}_{6}\right): 0.72$ (3H, $\mathrm{t}$, $\left.\mathrm{CH}_{2} \mathrm{CH}_{3}\right): 3.64\left(2 \mathrm{H}, \mathrm{q}, \underline{\mathrm{CH}}_{2} \mathrm{CH}_{3}\right) ; 3.60$ and $3.92\left(2 \mathrm{H}, \mathrm{d}\right.$ and d, J = $\left.15 \mathrm{~Hz}, \mathrm{SCH}_{2}\right) ; 4.80(1 \mathrm{H}, \mathrm{s}, 4-\mathrm{H})$; 7.62 and $8.22,7.72$ and $8.24\left(8 \mathrm{H}, \mathrm{d}\right.$ and $\mathrm{d}, \mathrm{d}$ and $\left.\mathrm{d}, 4,6-\mathrm{C}_{6} \mathrm{H}_{4}\right) 7.68$ and $7.96\left(2 \mathrm{H}\right.$, s and $\left.\mathrm{s}, \mathrm{CONH}_{2}\right)$; $11.10(1 \mathrm{H}, \mathrm{s}, \mathrm{NH})$. Anal. Calcd for $\mathrm{C}_{23} \mathrm{H}_{19} \mathrm{~N}_{5} \mathrm{O}_{7} \mathrm{~S}: \mathrm{C} 54.22, \mathrm{H} 3.76, \mathrm{~N} 13.75, \mathrm{~S} 6.29$. Found C 54.11, H $3.82, \mathrm{~N} 13,68, \mathrm{~S} 6.08$

In a similar manner (4-methoxybenzaldehyde was used instead of benzaldehyde) 2:carbamoylmethylthio-3-cyano-5-ethoxycarbonyl-4-(4-methoxyphenyl-6-(4-nitrophenyl)-1,4dihydropyridine $1 \mathrm{c}$ (yield $64 \%$ ) was obtained as slightly yellow powder; mp $186-189^{\circ} \mathrm{C}$. IR: 1686, $1708(\mathrm{C}=\mathrm{O}) ; 2198(\mathrm{C} \equiv \mathrm{N}) ; 3196,3304,3368\left(\mathrm{NH}, \mathrm{NH}_{2}\right) .{ }^{1} \mathrm{H}$ NMR (DMSO-d 6 ): $0.74(3 \mathrm{H}, \mathrm{t}$, $\left.\mathrm{CH}_{2} \mathrm{CH}_{3}\right)_{2} 3.60$ and $3.84\left(2 \mathrm{H}, \mathrm{d}\right.$ and d, J=15 Hz, $\left.\mathrm{SCH}_{2}\right) ; 3.72\left(2 \mathrm{H}, \mathrm{q}, \underline{\mathrm{CH}}_{2} \mathrm{CH}_{3}\right) ; 3.74\left(1 \mathrm{H}, \mathrm{s}, \mathrm{OCH}_{3}\right)$; $4.56(1 \mathrm{H}, \mathrm{m}, 4-\mathrm{H}) ; 4.94$ and $7.22\left(4 \mathrm{H}\right.$, br. $\mathrm{s}$ and br. s, $\left.4-\mathrm{C}_{6} \mathrm{H}_{4}\right) ; 7.58$ and $7.92(2 \mathrm{H}, \mathrm{br} . \mathrm{s}$, and br. $\mathrm{s}$, $\left.\mathrm{CONH}_{2}\right) ; 7.71$ and $8.27\left(4 \mathrm{H}\right.$, d and d, 6- $\left.\mathrm{C}_{6} \mathrm{H}_{4}\right) ; 10.86(1 \mathrm{H}, \mathrm{s}, \mathrm{NH})$. Anal. Calcd. for $\mathrm{C}_{24} \mathrm{H}_{22} \mathrm{~N}_{4} \mathrm{O}_{6} \mathrm{~S}: \mathrm{C}$ 58.30; H 4.50; N 11.30; S 6.50. Found: C 58.19; H 4.44; N 11.28, S 6.62.

In a similar manner (4-hydroxybenzaldehyde was used instead of benzaldehyde) 2 carbamovlmethylthio-3-cyano-5-ethoxycarbonyl-4-(4-hydroxyphenyl-6-(4-nitrophenyl)-1.4dihydropyridine $1 \mathrm{~d}$ (yield $70 \%$ ) was obtained as colourless powder; mp $219-221^{\circ} \mathrm{C}$. IR: 1666 , $1680(\mathrm{C}=\mathrm{O}) ; 2194(\mathrm{C} \equiv \mathrm{N}) ; 3184,3320,3430\left(\mathrm{NH}, \mathrm{NH}_{2}\right) .{ }^{1} \mathrm{H}$ NMR (DMSO-d $\left.{ }_{6}\right): 0.72(3 \mathrm{H}, \mathrm{t}$, $\left.\mathrm{CH}_{2} \mathrm{CH}_{3}\right) ; 3.62$ and $3.86\left(2 \mathrm{H}, \mathrm{d}\right.$ and $\left.\mathrm{d}, \mathrm{J}=15 \mathrm{~Hz}, \mathrm{SCH}_{2}\right) ; 3.72\left(2 \mathrm{H}, \mathrm{q}, \mathrm{CH}_{2} \mathrm{CH}_{3}\right) ; 4.58(1 \mathrm{H}, \mathrm{s}, 4-\mathrm{H})$; 6.72 and $7.10\left(4 \mathrm{H}, \mathrm{d}\right.$ and $\left.\mathrm{d}, 4-\mathrm{C}_{6} \mathrm{H}_{4}\right) ; 7.57$ and $7.90\left(2 \mathrm{H}\right.$, br. s, and br. s, $\left.\mathrm{CONH}_{2}\right), 7.68$ and $8.23(4 \mathrm{H}$, $\mathrm{d}$ and d, 6- $\left.\mathrm{C}_{6} \mathrm{H}_{4}\right) ; 9.36(\mathrm{IH}, \mathrm{s}, \mathrm{OH}) ; 10.80(1 \mathrm{H}, \mathrm{s}, \mathrm{NH})$. Anal. Calcd. for $\mathrm{C}_{23} \mathrm{H}_{20} \mathrm{~N}_{4} \mathrm{O}_{6} \mathrm{~S}: \mathrm{C} 57.49 ; \mathrm{H}$ 4.20, N 11.66; S 6.67. Found: C 57.34; H 4.25; N 11.59; S 6.72. 
2-Carbamoylmethylthio-3-cyano-5-ethoxycarbonvl-6-hydroxv-6-(4-nitrophenvl)-4-phenvl-1,

4, 5, 6-tetrahydropyridine $\underline{2}$. A mixture of ethyl 4-nitrobenzoylacetate (1.19 g, $5 \mathrm{mmol})$, benzaldehyde $(0.53 \mathrm{~g}, 5 \mathrm{mmol})$, cyanothioacetamide $(0.5 \mathrm{~g}, 5 \mathrm{mmol})$ and piperidine $(0.55 \mathrm{ml}, 5.5$ $\mathrm{mmol}$ ) in $25 \mathrm{ml}$ of ethanol was shortly heated until dissolution and stirred for $10 \mathrm{~min}$ at ambient temperature. Then to the reaction mixture iodoacetamide $(1.02 \mathrm{~g}, 5.5 \mathrm{mmol})$ was added, shortly heated and stirred for $1 \mathrm{~h}$ at the ambient temperature. The precipitate was filtered and washed with $10 \mathrm{ml}$ of ethanol, $10 \mathrm{ml}$ of water to give $1.62 \mathrm{~g} \mathrm{(67 \% )} \mathrm{of} 2$ as slightly yellow crystals; $\mathrm{mp} 179$ $180^{\circ} \mathrm{C}$. IR: $1676,1705(\mathrm{C}=\mathrm{O}) ; 2200(\mathrm{C} \equiv \mathrm{N}) ; 3164,3198,3328,3444,3472\left(\mathrm{NH}, \mathrm{NH}_{2}, \mathrm{OH}\right) .{ }^{1} \mathrm{H}$ NMR (DMSO-d $\left.\mathrm{d}_{6}\right): 0.45\left(3 \mathrm{H}, \mathrm{t}, \mathrm{CH}_{2} \mathrm{CH}_{3}\right) ; 3.04(1 \mathrm{H}, \mathrm{d}, \mathrm{J}=12 \mathrm{~Hz}, 5-\mathrm{H}) ; 3.44\left(2 \mathrm{H}, \mathrm{q}, \mathrm{CH}_{2} \mathrm{CH}_{3}\right): 3.62$ $\left(2 \mathrm{H}, \mathrm{s}, \mathrm{SCH}_{2}\right) ; 4.12(1 \mathrm{H}, \mathrm{d}, \mathrm{J}=12 \mathrm{~Hz}, 4-\mathrm{H}) ; 6.80(1 \mathrm{H}, \mathrm{s}, \mathrm{OH}), 7.25(5 \mathrm{H}, \mathrm{m}, 4-\mathrm{Ph}), 7.50$ and 7.90 $\left(2 \mathrm{H}, \mathrm{br} . \mathrm{s}\right.$ and br. s, $\left.\mathrm{CONH}_{2}\right) ; 7.86$ and $8.22\left(4 \mathrm{H}, \mathrm{d}\right.$ and d, 6- $\left.\mathrm{C}_{6} \mathrm{H}_{4}\right) ; 9.32(1 \mathrm{H}, \mathrm{s}, \mathrm{NH})$. Anal. Calcd. for $\mathrm{C}_{23} \mathrm{H}_{22} \mathrm{~N}_{4} \mathrm{O}_{6} \mathrm{~S}$ : C 57.25; H 4.60; N 11.61; S 6.64. Found: C 57.41, H 4.55, N 11.57; S 6.70.

Dehydrogenation of 6-hvdroxy-1, 4, 5, 6-tetrahydropyridine 2. 1, 4, 5, 6-Tetrahydropyridine $\underline{2}$ $(0.48 \mathrm{~g}, 1 \mathrm{mmol})$ in $5 \mathrm{ml}$ of $0.5 \mathrm{M} \mathrm{HCl}$-ethanol solution was shortly heated till dissolution and stirred at ambient temperature for $30 \mathrm{~min}$. The precipitate was filtered and washed with $5 \mathrm{ml}$ of ethanol to give $0.44 \mathrm{~g}(95 \%)$ of $\underline{\mathbf{1 a}}$ as yellow crystals; $\mathrm{mp} 208-210^{\circ} \mathrm{C}$.

\section{3-Amino-2-carbamovl-5-ethoxycarbonvl-6-(4-nitrophenyl)-4-phenylthieno[2,3-blpyridine 3a.}

A mixture of dihydropyridine $1 \mathbf{a}(0.93 \mathrm{~g}, 2 \mathrm{mmol})$ and $1 \mathrm{ml} 4 \mathrm{M} \mathrm{KOH}$ water solution in $20 \mathrm{ml}$ of ethanol was refluxed for $10 \mathrm{~min}$, stirred at ambient temperature for $30 \mathrm{~min}$. The precipitate was filtered and washed with $20 \mathrm{ml}$ of ethanol, $20 \mathrm{ml}$ of water to give $0.68 \mathrm{~g} \mathrm{(73 \% )} \mathrm{of} \mathbf{3 a}$ as yellow powder, mp $273-275^{\circ} \mathrm{C}$. IR: $1658,1716(\mathrm{C}=\mathrm{O})$; 3158, 3320, 3382, $3466\left(\mathrm{NH}_{2}\right)$; ${ }^{1} \mathrm{H}$ NMR (DMSO$\left.\mathrm{d}_{6}\right): 0.73\left(3 \mathrm{H}, \mathrm{tt}, \mathrm{CH}_{2} \mathrm{CH}_{3}\right) ; 3.81\left(2 \mathrm{H}, \mathrm{qq}, \mathrm{CH}_{2} \mathrm{CH}_{3}\right), 5.70\left(2 \mathrm{H}\right.$, br.s, 3- $\left.\mathrm{NH}_{2}\right) ; 7.3-8.5(11 \mathrm{H}$, complex, $4-\mathrm{C}_{6} \mathrm{H}_{5}, 6-\mathrm{C}_{6} \mathrm{H}_{4}$ and 2- $\mathrm{CONH}_{2}$ ). Anal. Calcd for $\mathrm{C}_{23} \mathrm{H}_{18} \mathrm{~N}_{4} \mathrm{O}_{5} \mathrm{~S}: \mathrm{C} 59.73, \mathrm{H} \mathrm{3.92,} \mathrm{N} \mathrm{12.11,}$ S 6.93. Found C 60.09, H 3.82, N 12.19, S 6.90.

In a similar manner by treatment dihydropyridine $\underline{\mathbf{1 b}}$ with $\mathrm{KOH}$ water solution in ethanol 3-amino-2-carbamovl-4,6-di-(4-nitrophenvl)-5-ethoxycarbonvlthieno[2,3-blpyridine $\underline{3 b}$ as orange powder was obtained, yield $80 \%, \mathrm{mp} 227-230^{\circ} \mathrm{C}$; IR: 1655, $1723(\mathrm{C}=\mathrm{O}) ; 3170,3322$, 3468, $3490\left(\mathrm{NH}_{2}\right)$; ${ }^{1} \mathrm{H}$ NMR (DMSO-d 6 ): $0.72\left(3 \mathrm{H}, \mathrm{t}, \mathrm{CH}_{2} \mathrm{CH}_{3}\right) .3 .82\left(2 \mathrm{H}, \mathrm{q}, \mathrm{CH}_{2} \mathrm{CH}_{3}\right) ; 5.52$ and $5.60\left(4 \mathrm{H}\right.$, br.s and br.s. $3-\mathrm{NH}_{2}$ and $\left.\mathrm{CONH}_{2}\right) ; 7.86$ and $8.32,7.87$ and $8.43(8 \mathrm{H}, \mathrm{d}$ and $\mathrm{d}$, $\mathrm{d}$ and $\mathrm{d}$, 4,6- $\mathrm{C}_{6} \mathrm{H}_{4}$ ). Anal. Calcd for $\mathrm{C}_{23} \mathrm{H}_{17} \mathrm{~N}_{5} \mathrm{O}_{7} \mathrm{~S}: \mathrm{C} 54.44, \mathrm{H} 3.38, \mathrm{~N} 13.80, \mathrm{~S} 6.32$. Found $\mathrm{C} 54.73, \mathrm{H} \mathrm{3.33}$, N 13.91, S 6.35. Found: C 54.17; H 3.20; N 13.60; S 6.17.

In a similar manner by treatment dihydropyridine $1 \mathrm{c}$ with $\mathrm{KOH}$ water solution in ethanol 


\section{3-amino-2-carbamovl-4-(4-methoxyphenyl)-6-(4-nitrophenyl)-5-ethoxycarbonyl-thieno [2,3-}

blpyridine $3 \mathrm{c}$ as orange powder was obtained, yield $70 \%$, mp $269-272^{\circ} \mathrm{C}$; IR: $1654,1729(\mathrm{C}=0)$; $3142,3320,3460\left(\mathrm{NH}_{2}\right)$; ${ }^{1} \mathrm{H}$ NMR (DMSO-d $\left.)_{6}\right): 0.78\left(3 \mathrm{H}, \mathrm{t}, \mathrm{CH}_{2} \underline{\mathrm{CH}}_{3}\right) ; 3.80\left(2 \mathrm{H}, \mathrm{q}, \mathrm{CH}_{2} \mathrm{CH}_{3}\right) ; 3.82$ $(3 \mathrm{H}, \mathrm{s}, \mathrm{OMe}) ; 5.80\left(2 \mathrm{H}\right.$, br.s $\left.3-\mathrm{NH}_{2}\right) ; 7.0-8.6\left(10 \mathrm{H}\right.$, complex, 4,6- $\mathrm{C}_{6} \mathrm{H}_{4}$ and $\left.\mathrm{CONH}_{2}\right)$. Anal. Calcd for $\mathrm{C}_{24} \mathrm{H}_{20} \mathrm{~N}_{4} \mathrm{O}_{6} \mathrm{~S}$ : C 58.53, H 4.O9, N 11.38, S 6.51. Found C 58.81, H 3.99, N 11.42. S 6.59.

In a similar manner by treatment dihydropyridine $\underline{\mathbf{1 d}}$ with $\mathrm{KOH}$ water solution in ethanol

\section{3-amino-2-carbamoyl-4-(4-hydroxyphenyl)-6-(4-nitrophenyl)-5-ethoxycarbonyl-thieno[2.3-}

blpyridine $\underline{\text { 3d }}$ as orange powder was obtained, yield $62 \%, \mathrm{mp} 276-278^{\circ} \mathrm{C}, \mathrm{IR}: 1646,1718(\mathrm{C}=\mathrm{O})$; $3326,3412,3430\left(\mathrm{OH}, \mathrm{NH}_{2}\right)$; ${ }^{1} \mathrm{H}$ NMR (DMSO-d 6 ): $0.84\left(3 \mathrm{H}, \mathrm{t}, \mathrm{CH}_{2} \mathrm{CH}_{3}\right): 3.86\left(2 \mathrm{H}, \mathrm{q}, \mathrm{CH}_{2} \mathrm{CH}_{3}\right)$; $6.10\left(2 \mathrm{H}\right.$, br.s $\left.3-\mathrm{NH}_{2}\right) ; 6.60$ and $6.97\left(4 \mathrm{H}, \mathrm{d}\right.$ and d, $\left.4-\mathrm{C}_{6} \mathrm{H}_{4}\right) ; 7.25\left(2 \mathrm{H}\right.$, br.s, $\left.\mathrm{CONH}_{2}\right) ; 7.90$ and 8.30 $\left(4 \mathrm{H}, \mathrm{d}\right.$ and d, 6- $\left.\mathrm{C}_{6} \mathrm{H}_{4}\right) ; 835(1 \mathrm{H}, \mathrm{s}, \mathrm{OH})$. Anal. Calcd.for $\mathrm{C}_{23} \mathrm{H}_{18} \mathrm{~N}_{4} \mathrm{O} \mathrm{O}_{6} \mathrm{~S}: \mathrm{C} 57.74, \mathrm{H} 3.79, \mathrm{~N} 11.71$, S 6.70. Found C 54.47, H 3.62, N 11.53, S 6.54.

\section{References}

1 A.A.Krauze, Yu.E.Pelcher, G.Ya.Dubur, Chem.Heterocycl.Comp. 20, 12, 1400 (1984)

2 A.A.Krauze, E.E.Liepinsh, Yu.E.Pelcher, G.Ya.Dubur, Chem. Heterocycl. Comp. 23, 1, 102 (1987)

3 A.A.Krauze, R.O.Vitolina, M.R.Romanova, G.Duburs, Khim.-Farm.Zh. (in Russian), 22, 955 (1988); CA 109: 204604d (1988)

4 A.A.Krauze, V.Garaliene, G.Duburs, Khim.-Farm.Zh. (in Russian), 26, 5, 40 (1992); CA 118: $38791 \mathrm{lb}(1993)$

5 A.A.Krauze, A.G.Odinecs, A.A.Verreva, S.K.Germane, A.Kozhuhov, G.Ya.Dubur, Khim.Farm.Zh. (in Russian), 25, 40 (1991); CA 115: 223418 (1991)

6 I.E.Kirule, A.A.Krauze, A.H.Velena, D.J.Antipova, G.J.Arnicane, I.A.Vucina, G.Ya.Dubur, Khim.-Farm.Zh. (in Russian), 26, 59 (1992); CA 119: 724678f (1993)

7 M.J.Rubio, C.Seoane, J.L. Soto, A.Susaseta, Liebigs Ann.Chem. 210 (1986)

8 A.A.Krauze, E.E.Liepinsh, Yu.E.Pelcher, Z.A.Kalme, G.Ya.Dubur, Chem.Heterocycl.Comp., 21, 1, 61 (1987).

9 A.G.Odinecs, D.A.Berzina, A.H.Velena, I.A.Vucina, A.A.Krauze, G.Ya.Duburs, A.A.Kimenis, Biological membrane and patalogy of cell (in Russian), 127 (1986); CA 106: 148956 (1987)

10 A.Krauze, G.Duburs. Heterocyclic Communications, 6, 3, 239 (2000) 